CAOS 2020. The 20th Annual Meeting of the International Society for Computer Assisted Orthopaedic Surgery

\title{
Preliminary Validation of a Patient Outcome Prediction Tool Relative to Surgeon Predictions of Patient Outcome
}

\author{
Joshua G Twiggs ${ }^{1}$, David Liu ${ }^{2}$, Justin Roe ${ }^{3}$, David Parker ${ }^{4}$ and Brad Miles ${ }^{1}$ \\ ${ }^{1} 360$ Knee Systems, Sydney, Australia \\ ${ }^{2}$ Gold Coast Centre for Bone \& Joint Surgery \\ ${ }^{3}$ North Sydney Orthopaedic and Sports Medicine Clinic, Sydney, Australia \\ ${ }^{4}$ Sydney Orthopaedic Research Institute Sydney, Australia
}

\begin{abstract}
Currently, patient selection for Total Knee Arthroplasties (TKA) is surgeon specific. A combination of patient reported symptoms, clinical examination findings, and radiological criteria are combined in an idiosyncratic fashion based on the surgeon's individual clinical experiences. Predictive models offer an alternative by providing more detailed information on expected patient outcome. This study presents validation relative to surgeon predictions of such a predictive model, the Patient Expectation Management (PEM) tool.

A cohort of patients undertook a survey covering the KOOS questionnaire and a number of other questions pertaining to comorbidities prior to their consultation. From this survey, a prediction of final state and assessment of current state was generated. Prior to seeing the prediction but after consulting the patient, surgeons were asked to score out of 100 a) their understanding of the patient's current pain state and b) their prediction of the patient's pain level following surgery. 35 of the patients were selected for TKA surgery and have gone on to have 12 month Knee Osteoarthritis and Outcome Scores (KOOS) captured.

The predicted change in the PEM predicted score (preop to postop difference) had a relatively high correlation with the actual KOOS pain improvement achieved $(r=0.71$, $\mathrm{p}<0.001)$, compared to no significant correlation for the surgeon prediction $(r=0.24$, $\mathrm{p}=0.20$ ). Significant correlations also existed for changes in KOOS symptoms score $(\mathrm{r}=0.70, \mathrm{p}<0.001)$ and KOOS Activities of Daily Living (ADL) score $(\mathrm{r}=0.42, \mathrm{p}=0.02)$.

This study showed that, compared to a set of surgeon predictions of outcome following a consultation with patients, a predictive analytics tool was able to outperform in terms of predicting the improvement patients are likely to report following TKA.
\end{abstract}




\section{Introduction}

Occurrence of patient dissatisfaction and suboptimal patient reported outcomes remain up to $20 \%$ in Total Knee Arthroplasty (TKA) [1, 2]. Previous studies have shown back pain and other affected joints [3-5]; pre-existing pain and functional state of the knee [6]; the patients socioeconomic status [7]; depression and anxiety [7-9]; pain catastrophising personality type [10] and self-efficacy of the patient[11] to contribute to this dissatisfaction. Additionally, unachievable or unmet patient expectations have been shown to predict patient dissatisfaction [12, 13].

Currently, patient selection for TKA is surgeon specific. A combination of patient reported symptoms, clinical examination findings, and radiological criteria are combined with a host of factors which each surgeon tends to apply in an idiosyncratic fashion based on their individual clinical experiences. Predictive models can potentially offer an alternative to selection criteria by providing more detailed information on expected patient outcome. Such models can further be used to set patient expectations to an appropriate level based on patient specific risk factors and in doing so reduce incidence of unmet expectations. This study presents validation relative to surgeon predictions of such a predictive model, the Patient Expectation Management tool [14].

\section{Methods}

A cohort of 100 patients was enrolled drawn from the practices of 4 consultant surgeons with greater than ten years' experience of performing TKAs. Patients were enrolled prior to their initial surgeon consultation. All patients undertook a survey covering the KOOS questionnaire and a number of other questions pertaining to comorbidities prior to their consultation. The survey was administered through an iPad-based web app. From this survey, a prediction of final state and assessment of current state was generated of a similar form to that shown in Figure 1. The predictive model used is a Tree Augmented Naive Bayes Network and has been previously published on [14].

Prior to seeing the prediction but after consulting the patient, surgeons were asked to score out of 100 a) their understanding of the patient's current pain state and b) their prediction of the patient's pain level following surgery. From the PEM, assessment of the current pain state and prediction of final pain state was also extracted. 35 of the 100 patients were selected for TKA surgery and have gone on to have 12-month Knee Osteoarthritis and Outcome Scores (KOOS) captured. KOOS scores postoperatively and the improvement from preoperative KOOS scores have been compared to the predictions for each from both the PEM and the surgeons. In addition, the presence of a 'significant' improvement of at least 40 points and the relative predictions for cohorts that did and did not achieve this prediction have been considered.

\section{Results}

For the 35 surgical patients, average age was $70.5 \pm 6.5$ years, and $57 \%$ (20) were female, representing a typical TKA population.

No significant correlation was found between the either the PEM or surgeon prediction of outcome and the actual postoperative KOOS pain score. However, the predicted change in the PEM predicted score (preop to postop difference) had a relatively high correlation with the actual KOOS pain 
improvement achieved ( $\mathrm{r}=0.71, \mathrm{p}<0.001)$, compared to no significant correlation for the surgeon prediction $(\mathrm{r}=0.24, \mathrm{p}=0.20)$. Significant correlations also existed for changes in KOOS symptoms score $(\mathrm{r}=0.70, \mathrm{p}<0.001)$ and KOOS Activities of Daily Living (ADL) score $(\mathrm{r}=0.42, \mathrm{p}=0.02)$.

When segmenting into those who achieved a threshold of 40 points improvement in KOOS pain, the surgeon predictions failed to significantly differ, with the portion that improved receiving a prediction of 57 points compared to 49 for the portion that did not improve $(\mathrm{p}=0.25)$. The PEM, by comparison, predicted a 43 point improvement for those who did improve, and a 17 point improvement for those who did not, and the difference was significant $(\mathrm{p}<0.001)$. Overall, the actual improvement was, on average, 39.6 points; surgeons predicted 54.5 points and the PEM predicted 31.6.

\section{Discussion}

There is a clear need for shared decision-making tools to aid in selection of patients for TKA surgery. Historical focus on surgical accuracy and technique as the primary driver of outcomes has not solved the dissatisfaction of patients. Use of fixed criteria in selection for surgery such as PROMs scores [15] and radiology [16] may not be applicable in a range of patients due to the multiple factors that affect outcome and the complex interactions between them. The existence of predictive analytics tools such as this, used in concert with surgeons, represent a significant step forward in appropriate selection and expectation setting for TKA patients

Significantly, the surgeon predictions were consistently optimistic of actual improvements, while the PEM's predicted improvements were pessimistic, but closer to the actual achieved. It may be that this innate 'pessimism' is required to unlock the predictive capability of the system.

\section{Conclusion}

This study showed that, compared to a set of surgeon predictions of outcome following a consultation with patients, a predictive analytics tool was able to outperform in terms of predicting the improvement patients are likely to report following TKA. Such tools are justified for use alongside experienced surgeons in preoperative decision to operate and patient expectation setting. 


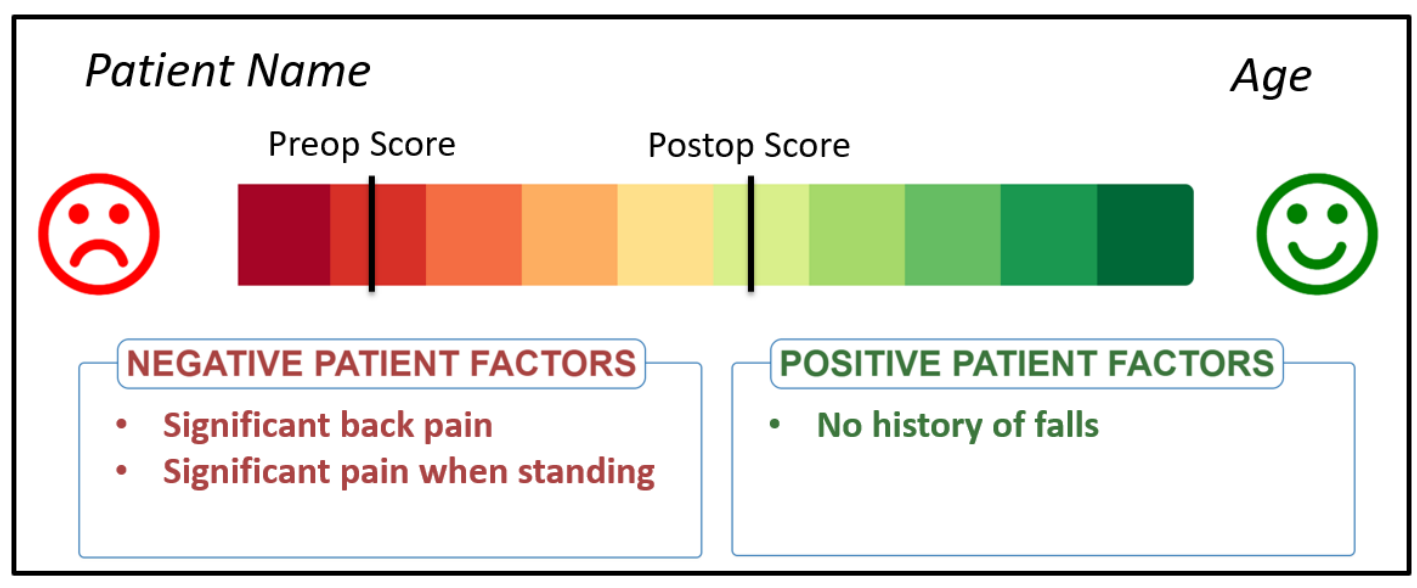

Figure 1: Interface created for use in the patient consultation. 3 elements on the interface are defined: a preoperative reference state; a postoperative prediction; and a set of boxes detailing positive and negative prediction factors. The live version incorporates a toggle switch to jump between preoperative position and postoperative prediction.

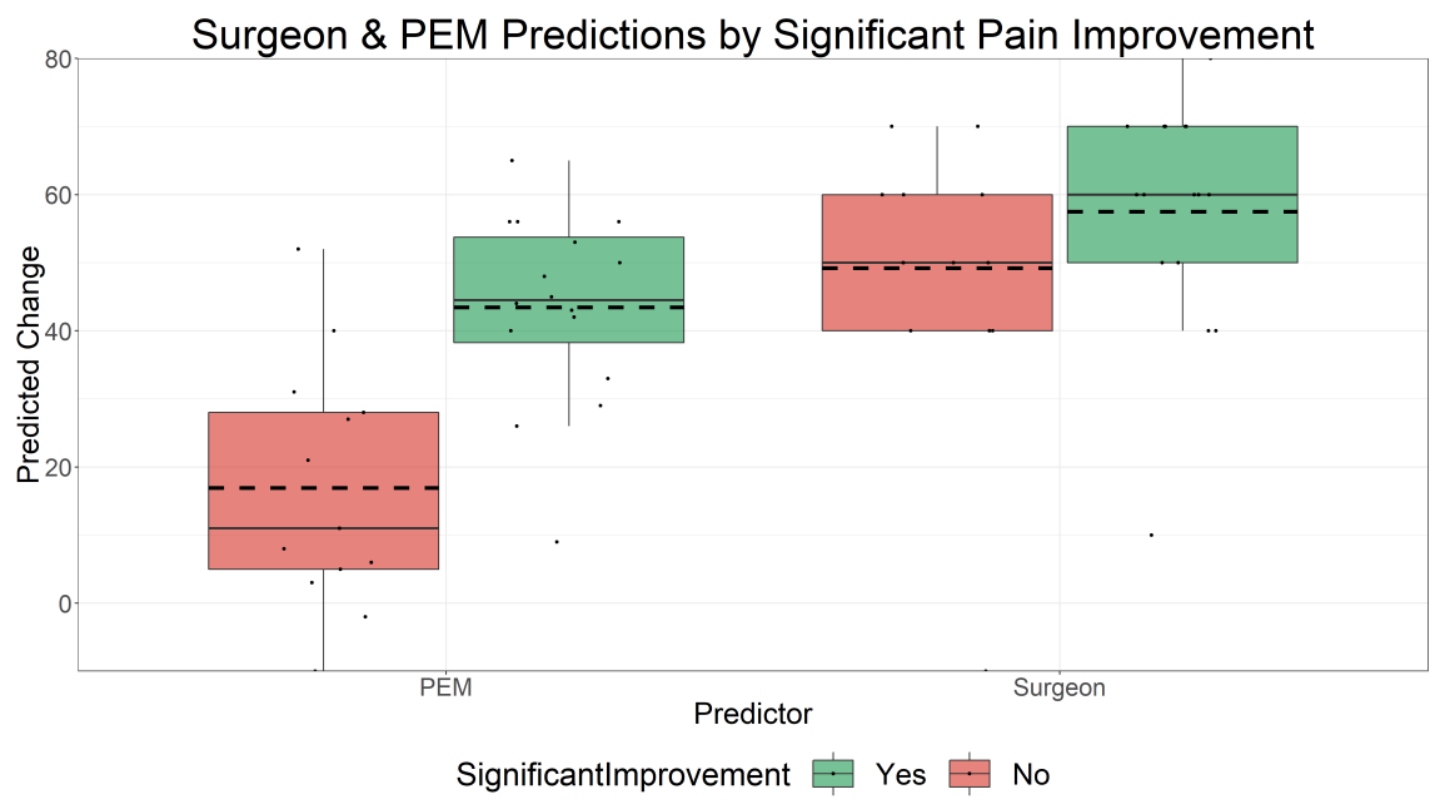

Figure 2. Comparison of PEM and Surgeon predictions of improvement in KOOS pain score, separated by actual patient score improvement following surgery. Notably, overall predictions with the PEM are more negative than surgeon predictions, but it demonstrates improved capability to differentiate between patients less and more likely improve dramatically. 


\section{References}

1. Bourne, R.B., et al., Patient satisfaction after total knee arthroplasty: who is satisfied and who is not? Clin Orthop Relat Res, 2010. 468(1): p. 57-63.

2. Beswick, A.D., et al., What proportion of patients report long-term pain after total hip or knee replacement for osteoarthritis? A systematic review of prospective studies in unselected patients. BMJ Open, 2012. 2(1): p. e000435.

3. Escobar, A., et al., Effect of patient characteristics on reported outcomes after total knee replacement. Rheumatology (Oxford), 2007. 46(1): p. 112-9.

4. Scott, C.E., et al., Predicting dissatisfaction following total knee replacement: a prospective study of 1217 patients. J Bone Joint Surg Br, 2010. 92(9): p. 1253-8.

5. $\quad$ Clement, N.D., et al., Total knee replacement in patients with concomitant back pain results in a worse functional outcome and a lower rate of satisfaction. Bone Joint J, 2013. 95-B(12): p. 1632-9.

6. $\quad$ Baker, P.N., et al., The role of pain and function in determining patient satisfaction after total knee replacement. Data from the National Joint Registry for England and Wales. J Bone Joint Surg Br, 2007. 89(7): p. 893-900.

7. Judge, A., et al., Predictors of outcomes of total knee replacement surgery. Rheumatology (Oxford), 2012. 51(10): p. 1804-13.

8. Brander, V.A., et al., Predicting total knee replacement pain: a prospective, observational study. Clin Orthop Relat Res, 2003(416): p. 27-36.

9. Wylde, V., et al., Acute postoperative pain at rest after hip and knee arthroplasty: severity, sensory qualities and impact on sleep. Orthop Traumatol Surg Res, 2011. 97(2): p. 139-44.

10. Sullivan, M., et al., Psychological determinants of problematic outcomes following Total Knee Arthroplasty. Pain, 2009. 143(1-2): p. 123-9.

11. Wylde, V., S. Dixon, and A.W. Blom, The role of preoperative self-efficacy in predicting outcome after total knee replacement. Musculoskeletal Care, 2012. 10(2): p. 110-8.

12. Noble, P.C., et al., The John Insall Award: Patient expectations affect satisfaction with total knee arthroplasty. Clin Orthop Relat Res, 2006. 452: p. 35-43.

13. Sullivan, M., et al., The role of presurgical expectancies in predicting pain and function one year following total knee arthroplasty. Pain, 2011. 152(10): p. 2287-93.

14. Twiggs, J.G., et al., Clinical and Statistical Validation of a Probabilistic Prediction Tool of Total Knee Arthroplasty Outcome. J Arthroplasty, 2019.

15. Clement, N.D., D. Macdonald, and R. Burnett, Predicting patient satisfaction using the Oxford knee score: where do we draw the line? Arch Orthop Trauma Surg, 2013. 133(5): p. 689-94.

16. Schnurr, C., et al., Pre-operative arthritis severity as a predictor for total knee arthroplasty patients' satisfaction. Int Orthop, 2013. 37(7): p. 1257-61. 